\title{
Theoretical Estimation of Non Linear Optical Properties of Organic Framework Containing $p$-Nitroaniline Linked to Naphthalene/azanaphthalene Through Saturated Carbon Chain
}

\author{
ANJU LINDA VARGHESE ${ }^{1 *}$ and M. GEORGE ${ }^{2}$ \\ ${ }^{1}$ Department of Chemistry, Catholicate College, Pathanamthitta-689 645, India \\ ${ }^{2}$ Department of Chemistry, Sacred Heart College (Autonomous), Thevara, Kochi-682013, India \\ lindaanju@gmail.com
}

Received 12 April 2018 / Accepted 28 April 2018

\begin{abstract}
Nonlinear optical (NLO) properties of $N$-substituted $p$-nitroaniline molecules, $N$-[3(naphthalene-1-yloxy)butyl]-4-nitroaniline (1), $\quad N$-[3-(2,4,5-triazanaphthalene-1-yloxy)butyl]-4-nitroaniline (2) and $N$-[3-(2,4,5,7-tetraazanaphthalene-1-yloxy)butyl]-4-nitroaniline (3) have been calculated theoretically. The theoretical calculations were performed with three different hybrid density functional theories (DFT) i.e. BPV86, B3LYP and M-06 with 6-31++G (d,p) basis set. These molecular systems exhibit large first static hyperpolarizabilities. The optical response of these molecular systems is compared with that of $p$-nitroaniline, which is an excellent NLO dye. The result shows that, naphthalene or azanaphthalene derivatives of $p$-nitroaniline, exhibit large hyperpolarizability than $p$-nitroaniline and hence can be recommended for photovoltaic device fabrication.
\end{abstract}

Keywords: NLO, Hyperpolarizability, BPV86, B3LYP and M-06

\section{Introduction}

Molecular materials with nonlinear optical (NLO) properties are currently attracting considerable attention because of their potential applications in information technology and industry. Theoretical calculations play a significant role in understanding the polarization mechanism of NLO materials and their relation to structural characteristics. Last decade witnessed the development of new nonlinear optical materials of inorganic, organic and semi-organic types. Organic nonlinear optical materials have potentially high nonlinearities and rapid response to light ${ }^{1-4}$. They offer high degree of synthetic flexibility to tune their optical properties through structural modification ${ }^{5}$. The structure of organic NLO materials is based on the $\pi$-bond system extended over a large length scale of the molecule, which can be easily manipulated by substitution of electron donating and electron with drawing groups 
around the aromatic moieties, thus leading to an increased optical non-linearity ${ }^{6-8}$. The large value of the first hyperpolarizability, $\beta$, which is the measure of the nonlinear optical activity, is attributed to intramolecular charge transfer, which occurs due to the movement of electron cloud from electron donor to acceptor groups through a $\pi$ conjugated framework $^{9-12}$. The designing of new nonlinear optical materials relies heavily on the theoretical prediction of accurate electro-optical properties for aforementioned systems.

$p$-nitroaniline is one of the simplest molecules having electron donor and acceptor system connected by an aromatic ring and shows remarkable solvatochromism and large NLO properties ${ }^{13,14}$. NLO studies on $N$-substituted $p$-nitroaniline are being done to improve its nonlinear response ${ }^{15,16}$. The ground state geometrical energy, dipole moment, polarizability and first static hyperpolarizability of $p$-nitroaniline (PNA), 2-methyl-4nitroaniline (MNA), $N$-methyl-4-nitroaniline (HMNA), $N$-methyl-2-methyl-4-nitroaniline (HMMNA), 4-nitrodimethylaniline (NDMA) and $N$-(4-nitrophenyl)- $N$-methylaminoacetonitrile (NPAN) has been investigated by density functional theory (DFT) using B3LYP/6-311G* basis set. The calculation results revealed that hyperpolarizability and its related properties were enhanced in various degrees upon successive substitution of alkyl groups at amino group of the PNA molecule ${ }^{17}$.

Also the polarizability of organic frameworks containing naphthalene has been extensively studied with different theoretical methods and is found to have good nonlinear response. An efficient method to modulate electron density distribution in this conjugated system is the incorporation of functional groups (spacers) into its backbone. Such studies are done on conjugated oligomers and polymers ${ }^{18,19}$. But investigations on donor-acceptor systems containing naphthalene or azanaphthalene linked to $N$-substituted $p$-nitroaniline through spacer groups in the backbone have not been reported in the literature. To the best of our knowledge, the present study is the first ever DFT calculation of NLO properties of $N$-substituted $p$-nitroaniline in which naphthalene and azanaphthalene linked through a saturated carbon chain as the $N$-substituent in gas phase.

The principal aim of this work is to undertake an exhaustive theoretical investigation on the structural, electronic and optical properties of naphthalene/azanaphthalene framework linked to $p$-nitroaniline through a spacer groups. The molecules with large hyperpolarizability values can be developed into NLO materials which are having potential applications in the optoelectronic devices of telecommunications, information storage, optical switching and photovoltaic devices like solar cells.

The molecules chosen for the present study are $N$-[3-(naphthalene-1-yloxy)butyl]-4nitroaniline (1), $\mathrm{N}$-[3-(2,4,5-triazanaphthalene-1-yloxy)butyl]-4-nitroaniline (2) and $\mathrm{N}$-[3(2,4,5,7-tetraazanaphthalene-1-yloxy)butyl]-4-nitroaniline (3). The structural details are presented in Figure 1 below.<smiles>[R]Oc1cccc2ccccc12</smiles>

1<smiles>[R]Oc1ncnc2ncccc12</smiles>

2<smiles>[R]Oc1ncnc2ncncc12</smiles>

3<smiles>[R]#CC(C)CCNc1ccc([N+](=O)[O-])cc1</smiles>

Figure 1. Structures of molecules 1-3 


\section{Computational details}

Gaussian 09 software package was used for DFT calculation and calculations were performed at three different hybrid density functional theories (DFT) i.e. BPV86, B3LYP and $\mathrm{M}-06$ and $6-31++\mathrm{G}(\mathrm{d}, \mathrm{p})$ basis set was used. The ground state structures were optimized and frequency calculations were performed to ensure that the optimized structures are minima in the potential energy surface. HOMO and LUMO for all the molecules are identified. Gauss View 5 software was used for generating the input file and visualization of the results. The calculation were done using S20D300 workstation computer equipped with Intel 7 core processor and 24 GB RAM and Microsoft Windows as the operating system. Electric dipole moment, linear polarizability and first hyperpolarizability tensor components for the studied compounds were calculated by DFT approach which is currently one of the ultimate procedures for obtaining numerically accurate NLO responses.

\section{Results and Discussion}

\section{Geometry optimization}

The optimized molecular geometry represents an isolated molecule under ideal conditions with a stationary point at the potential energy surface. The convergence was confirmed by observing no imaginary vibrational frequencies.

Several conformational isomeric cisoid and transoid structures of molecules 1- 3 were optimized at B3LYP/6-31++G(d,p) level. The lowest energies of the cisoid and transoid conformations of three molecules are given in Figure 2 and their energies are enlisted in Table 1. The lowest energy transoid conformation of $\mathbf{1}$ is $10.75 \mathrm{KJ} / \mathrm{Mol}$ less in energy than the cisoid conformation. But the conversion of transoid to cisoid involves a rotation of $\mathrm{C}-\mathrm{O}$ bond of the naphthyl ether, require much higher energy, $36.70 \mathrm{~kJ} / \mathrm{mol}$. Hence transoid conformation is the preferred structure of 1.The optimized lowest energy structure of cisoid conformation of $\mathbf{2}$ is given in Figure 1. The lowest energy cisoid conformation is $5.48 \mathrm{~kJ} / \mathrm{mol}$ less in energy than the transoid conformation. When the chain length and consequently the flexibility of the molecule increased, the cisoid structure is more stable although the difference in energy is very small. DFT optimizations of the conformational isomers of $\mathbf{3}$ showed that the lowest energy conformation is the cisoid conformation.

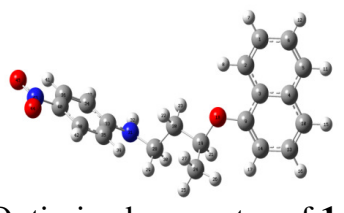

Optimized geometry of $\mathbf{1}$

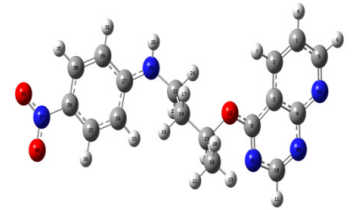

Optimized geometry of 2

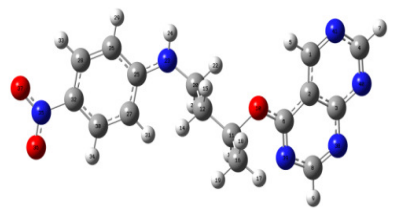

Optimized geometry of $\mathbf{3}$

Figure 2. Optimized geometries of $\mathbf{1}$ - $\mathbf{3}$

Table 1. Total energy and HOMO-LUMO gaps of molecules 1-3

\begin{tabular}{llcccccc}
\hline \multirow{2}{*}{ Compound } & Total energy & Difference & HOMO & \multicolumn{2}{c}{ LUMO } & \multicolumn{2}{c}{ HLG } \\
\cline { 2 - 7 } & Hartrees & kJ/Mol & Hartrees & Hartrees & Hartrees & $\mathrm{eV}$ \\
\hline \multirow{2}{*}{1} & Cisoid & -1108.90436 & 10.75 & -0.21101 & -0.09895 & 0.11206 & 3.04 \\
& Transoid & -1108.90846 & & -0.20422 & -0.07029 & 0.10397 & 3.64 \\
2 & Cisoid & -1157.07247 & \multirow{2}{*}{5.48} & -0.22343 & -0.07484 & 0.14859 & 4.04 \\
& Transoid & -1157.07038 & & -0.22519 & -0.07327 & 0.15192 & 4.13 \\
3 & Cisoid & -1173.12009 & \multirow{2}{*}{6.04} & -0.22564 & -0.08870 & 0.13694 & 3.72 \\
& Transoid & -1173.11779 & & -0.22722 & -0.08589 & 0.14133 & 3.85 \\
\hline
\end{tabular}


Analysis of the optimized geometries derived the following observation regarding the structure. The naphthalene part is coplanar with spacer group while the nitrophenyl part of the framework is twisted with respect to the spacer. This conclusion is supported by the relevant torsional angles listed (c.f. Table 5).

\section{Frontier molecular orbitals (FMOs)}

The energies of Frontier molecular orbitals viz. HOMO and LUMO, are helpful in investigating the electrical and chemical properties of substrates ${ }^{20}$. Table 2 depicts the HOMO and LUMO orbitals for 1-3 obtained using B3LYP functional and 6-31++G(d,p) basis set. HOMO of $\mathbf{1}$ extends over naphthalene donor moieties, whereas it's LUMO resides over nitro phenyl part. On the other hand HOMO and LUMO of both $\mathbf{2}$ and $\mathbf{3}$ are identical. HOMO of them extends over nitrophenyl group and saturated carbon chain whereas LUMO resides over azanaphthalene ring.

Table 2. HOMO and LUMO orbitals for 1 - 3

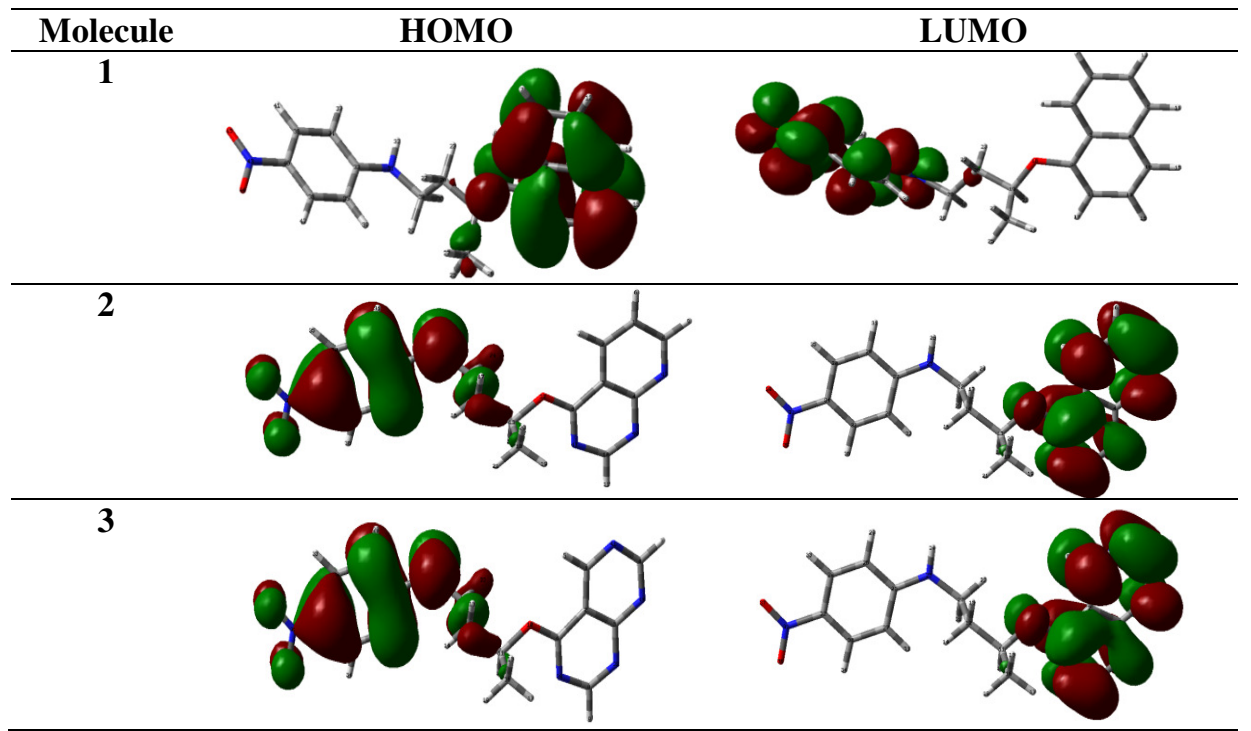

Using the ground state HOMO and LUMO energies, the chemical hardness $(\eta)$, the electronic chemical potential $(\mu)$ and the global electrophilicity index $(\omega)$ have been estimated and the calculated values are listed in Table 3.

\section{Chemical reactivity}

The kinetic stability of aforementioned compounds can be monitored by the HOMO-LUMO energy gaps ${ }^{21}$. Smaller HOMO-LUMO energy gap leads to lower kinetic stability and higher chemical reactivity. All our results showed that $\mathbf{2}$ is much more reactive than $\mathbf{1}$. The chemical reactivity description parameter like the chemical hardness $(\eta)$ is useful in studying the stability and reactivity of compounds. It is given in terms of the energies of the HOMOs and LUMOs ${ }^{22}$;

$$
\eta=\left(\frac{E_{H O M O}-E_{L U M O}}{2}\right)
$$


This formula indicates that soft compounds have small chemical hardness, while hard ones have large chemical splitting. The electronic chemical potential $(\mu)$ shows the escaping tendency of electrons in compounds ${ }^{23}$ and given by Equation $(2)^{24,25}$;

$$
\mu=-\left(\frac{E_{\text {HOMO }}+E_{L U M O}}{2}\right)
$$

The global electrophilicity index $(\omega)$ estimates the stabilizing energy when a surrounding environment supplies a chemical entity with an additional electronic charge. The index $(\omega)$ relates to the electronic chemical potential $(\mu)$ and the chemical hardness $(\eta)$ through Equation (3) ${ }^{23}$;

$$
\omega=\frac{\mu^{2}}{2 \eta}
$$

The values of the global electrophilicity indexes have been calculated and presented in Table 3. The order of electrophilicity index is $3>2>1$. It is evident from the table that $\mathbf{1}$ is the stronger nucleophile than $\mathbf{2}$ and 3. It implies that electron density over $\mathbf{1}$ becomes more polarized towards external optical response. Consequently the degree of delocalization increases which results in higher hyperpolarizability.

Table 3. Ground state HOMO and LUMO energies, the chemical hardness $(\eta)$, the electronic chemical potential $(\mu)$ and the global electrophilicity index $(\omega)$ of 1-3

\begin{tabular}{ccccccc}
\hline Compound & HOMO (a.u.) & LUMO (a.u.) & $\mathrm{E}_{\mathrm{g}}(\mathrm{eV})$ & $\eta$ & $\mu$ & $\omega$ \\
\hline $\mathbf{1}$ & -0.20422 & -0.07029 & 3.64 & 1.82 & 3.73 & 3.83 \\
$\mathbf{2}$ & -0.22343 & -0.07484 & 4.04 & 2.02 & 4.05 & 4.07 \\
$\mathbf{3}$ & -0.22564 & -0.08870 & 3.72 & 1.86 & 4.27 & 4.92 \\
\hline
\end{tabular}

\section{Optical activity}

DFT method with $6-31++\mathrm{G}(\mathrm{d}, \mathrm{p})$ basis set is selected for NLO calculations. Optical properties such as dipole moment, isotropic linear polarizability $\langle\alpha\rangle$, anisotropic linear polarizability $\Delta \alpha$ and first order or static hyperpolarizability $\beta$ were calculated using different functional BPV86, B3LYP and M-06.

The nonlinear optical response of an isolated molecule in an electric field $E_{i}$ can be presented as a Taylor series expansion of the total dipole moment, $\mu_{\mathrm{tot}}$, induced by the field:

$$
\begin{aligned}
& \mu_{\lambda}=\left\langle\psi(E)\left|\hat{\mu}_{\lambda}\right| \psi(E)\right\rangle \\
& \mu_{\lambda}=\mu_{\lambda}^{0}+\alpha_{\lambda \sigma} E_{\sigma}+\frac{1}{2 !} \beta_{\lambda \sigma v} E_{\sigma} E_{v}+\frac{1}{3 !} \gamma_{\lambda \sigma \nu \rho} E_{\sigma} E_{v} E_{\rho}+\ldots \ldots \ldots,
\end{aligned}
$$

Where, $\alpha$ is the linear polarizability, $\mu_{0}$ the permanent dipole moment and $\beta$ is the first hyperpolarizability tensor components. The isotropic (or average) linear polarizability and anisotropy of polarizability is defined $\operatorname{as}^{26,27}$;

Isotropic linear polarizability, $\langle\alpha\rangle=\frac{1}{3}\left(\alpha_{x x}+\alpha_{y y}+\alpha_{z z}\right)$

Anisotropic linear polarizability, $\Delta \alpha=\frac{1}{2}\left[\left(\alpha_{x x}-\alpha_{y y}\right)^{2}+\left(\alpha_{x x}-\alpha_{z z}\right)^{2}+\left(\alpha_{y y}-\alpha_{z z}\right)^{2}\right]^{1 / 2}$ 
The complete equation for calculating the total static first hyperpolarizability magnitude of Gaussian output is given as follows

First order hyperpolarizability,

$$
\beta_{t o t}=\left[\left(\beta_{x x x}+\beta_{x y y}+\beta_{x z z}\right)^{2}+\left(\beta_{y y y}+\beta_{y z z}+\beta_{y x x}\right)^{2}+\left(\beta_{z z z}+\beta_{z x x}+\beta_{z y y}\right)^{2}\right]^{1 / 2}
$$

The study involves the initial determination of dipole moment, isotropic linear polarizability, anisotropic linear polarizability and static hyperpolarizibilities in the gas phase. The results are compiled in Table 4.

Table 4. Dipole moment, isotropic linear polarizability, anisotropic linear polarizability and static hyperpolarizibilities of $\mathbf{1}$ - $\mathbf{3}$ using different levels of theory

\begin{tabular}{ccccc}
\hline Compound & Parameter & B3LYP & BPV86 & M-06 \\
\cline { 2 - 4 } $\mathbf{1}$ & Dipole Moment & 6.67 & 6.67 & 6.81 \\
& $<\alpha>$ & 232.69 & 242.27 & 242.82 \\
& $\Delta \alpha$ a.u & 268.37 & 289.77 & 292.27 \\
& $\beta_{\text {tot }}$ a.u. & 2306.04 & 2474.84 & 2499.03 \\
$\mathbf{2}$ & Dipole Moment & 7.68 & 7.41 & 7.53 \\
& $<\alpha>$ & 246.89 & 256.34 & 256.81 \\
& $\Delta \alpha$ a.u & 209.43 & 228.12 & 230.35 \\
& $\beta_{\text {tot }}$ a.u. & 2026.33 & 2223.51 & 2271.07 \\
$\mathbf{3}$ & Dipole Moment & 5.68 & 5.69 & 5.77 \\
& $<\alpha>$ & 225.93 & 235.61 & 236.22 \\
& $\Delta \alpha$ a.u & 186.68 & 207.31 & 210.093 \\
& $\beta_{\text {tot }}$ a.u. & 1963.41 & 1948.87 & 1938.16 \\
\hline
\end{tabular}

Results of the calculations showed that all three molecules show large first hyperpolarizability and consequently have good nonlinear response. Hyperpolarizability of $\mathbf{1}$ is greater than both $\mathbf{2}$ and $\mathbf{3}$. The magnitudes of hyperpolarizibilities depend on chemical reactivity and structural characteristics of the molecule. Degree of electron delocalization between the two rings, naphthalene and nitrophenyl, has an important role in increasing the hyperpolarizibilities. Optical response properties are governed by the increasing of both conjugation length and strength of donor and acceptor groups, planarity of the rings with spacer. Degree of electron delocalization between the two rings can be monitored by measuring effective distance between relevant atoms of the two rings (C7-C15) and coplanarity can be assessed by the torsional angles between the planes of the donor and acceptor subunits (angle between C11-O1-C7-C8 and angle between C17-C15-N1-C14). The aforementioned geometrical parameters obtained from the optimized geometries like torsional angles and effective distance between two rings are enlisted in Table 5.

From this table, it is evident that the effective distance between two rings for the above 3 molecules varies as $\mathbf{1}>\mathbf{2}>\mathbf{3}$. As the effective distance increases, it is believed that the extent of delocalization increases. So 1 is having large $\beta$ values than $\mathbf{2}$ and $\mathbf{3}$. The order of hyperpolarizability is as follows $\mathbf{1}>\mathbf{2}>\mathbf{3}$.

Table 5. Comparison of first hyperpolarizability for compounds 1-3 using B3LYP, BPV86 and M-06 with some selected angle and torsional angles

\begin{tabular}{ccccccc}
\hline & $\beta(\mathrm{B} 3 \mathrm{LYP})$ & $\beta(\mathrm{BPV} 86)$ & $\beta(\mathrm{M}-06)$ & $\mathrm{C}_{7}-\mathrm{C}_{15}(\AA) \mathrm{C}_{17}-\mathrm{C}_{15}-\mathrm{N}_{1}-\mathrm{C}_{14}\left({ }^{\circ}\right) \mathrm{C}_{11}-\mathrm{O}_{1}-\mathrm{C}_{7}-\mathrm{C}_{8}\left(^{\circ}\right)$ \\
\hline 1 & 2306.046 & 2474.841 & 2499.029 & 6.89 & -6.3 & 1.679 \\
2 & 2026.33 & 2223.519 & 2271.065 & 6.435 & -7.2 & 2.434 \\
3 & 1963.409 & 1948.879 & 1938.155 & 6.424 & -7.9 & 2.884 \\
\hline
\end{tabular}




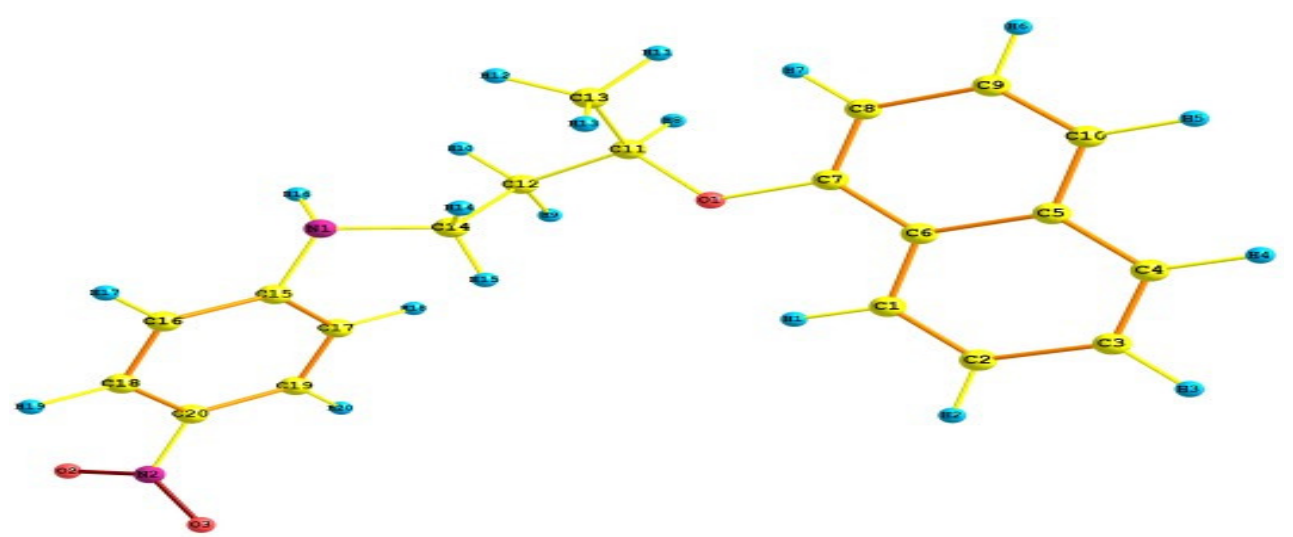

Figure 3. An atom numbering scheme of $\mathbf{1}$. The carbon, nitrogen, oxygen and hydrogen atoms are indicated by yellow, pink, red and blue colours, respectively

DFT studies on NLO properties of $p$-nitroaniline have been done at B3LYP and M06 using $6-31++\mathrm{G}(\mathrm{d}, \mathrm{p})$ basis set. The dipole moment, isotropic linear polarizability, anisotropic linear polarizability and static hyperpolarizibilities of $p$-nitroaniline is presented in Table 6 .

Table 6. Dipole moment, isotropic linear polarizability, anisotropic linear polarizability and static hyperpolarizibilities of $p$-nitroaniline using different levels of theory

\begin{tabular}{cccc}
\hline Compound & Parameter & B3LYP & M-06 \\
\hline & Dipole Moment & 7.1564 & 7.2456 \\
$p$-nitroaniline & $<\alpha>$ & 86.35078 & 86.09924 \\
& $\Delta \alpha$ a.u & 134.1624 & 132.8782 \\
& $\beta_{\text {tot }}$ a.u. & 1254.977 & 1327.441 \\
\hline
\end{tabular}

It is clear that hyperpolarizability of $p$-nitroaniline can be improved by adding substituent at $N$-terminal and hence those molecular frameworks can be considered as good NLO dyes and have potential applications in frequency conversion, image processing, data storage, fiber optic communication etc.

\section{Conclusion}

Nonlinear optical (NLO) properties of $N$-substituted $p$-nitroaniline molecules, $N$-[3(naphthalene-1-yloxy)butyl]-4-nitroaniline, $N$-[3-(2,4,5-triazanaphthalene-1-yloxy)butyl]-4nitroaniline and $N$-[3-(2,4,5,7-tetraazanaphthalene-1-yloxy)butyl]-4-nitroaniline have been calculated theoretically using three different hybrid density functional theories (DFT) i.e. BPV86, B3LYP and M-06 with 6-31++G(d,p) basis set. The results showed that these molecular systems have large first static hyperpolarizabilities. Their hyperpolarizibilities are compared with that of $p$-nitroaniline which is an excellent NLO dye and it is found to be higher. The order of hyperpolarizability of these $3 \mathrm{~N}$-substituted $p$-nitroaniline molecules are 1 $>2>3$. This can be attributed to their chemical reactivity and structural characteristics. Firstly, $\mathbf{1}$ is more nucleophilic than $\mathbf{2}$ and $\mathbf{3}$ and hence there is a greater delocalization. Secondly, the extent of delocalization has been reaffirmed by the effective distance between two rings, which is greater for $\mathbf{1}$. Finally, naphthalene ring of compound $\mathbf{1}$ is more coplanar to spacer group than $\mathbf{2}$ and $\mathbf{3}$, which enhances the delocalization. Hyperpolarizability of these molecules was compared with that of $p$-nitroaniline which shows remarkable NLO property. It is revealed that substitution of naphthalene or azanaphthalene linked through a 
spacer group at the amino terminal of $p$-nitroaniline tremendously increases its non linear optical properties. Thus the above three $N$-substituted $p$-nitroaniline show improved NLO properties than $p$-nitroaniline and can have potential application in the field of optoelectronics.

\section{References}

1. Ambujam K, Rajarajan K, Selvakumar S, Madhavan J, Gulam Mohamed and Sagayaraj P, Opt Mater., 2007, 29(6), 657-662; DOI:10.1016/j.optmat.2005.11.008

2. Meera K., Muralidharan R, Dhanasekaran R., Prapun Manyum and Ramasamy P, $J$ Cryst Growth, 2004, 263(1-4), 510-516; DOI:10.1016/j.jcrysgro.2003.11.093

3. Bredas J L, Adant C, Tackx P, Persoons A and Pierce B M, Chem Rev., 1994, 94(1), 243-278; DOI: $10.1021 / \mathrm{cr} 00025 \mathrm{a} 008$

4. Dalton L R, Harper A W, Ghosn R, Steier W H, Ziari M, Fetterman H, Shi Y, Mustacich R V, Jen A K Y and Shea K J, Chem Mater., 1995, 7(6), 1060-1081; DOI:10.1021/cm00054a006

5. Justin Raj C, Dinakaran S, Krishnan S, Miltonboaz B, Robert R and Jerome Das S, Opt Commun., 2008, 281(8), 2285-2290; DOI:10.1016/j.optcom.2007.12.019

6. Nalwa H S and Miyata S, Nonlinear Opt Org Mol Polym., 1997, 45, 611-617;

7. Shanthi A, Krishnan C and Selvarajan P, J Cryst Growth, 2014, 393, 7-12; DOI:10.1016/j.jcrysgro.2013.12.011

8. Sethuram M, Bhargavi G, Rajasehakaran M V, Dhandapani M and Amirthaganesan G, Optik., 2014, 125(1), 55-60; DOI:10.1016/j.ijleo.2013.06.069

9. Kulhanek J and Bures F, J Org Chem., 2012, 8, 25-49; DOI:10.3762/bjoc.8.4

10. Kim T D and Lee K S, Macromol Rapid Commun., 2015, 36(11), 943-58; DOI:10.1002/marc.201400749

11. Chen X, Jia C, Wan Z, Zhang J and Yao X, Spectrochim Acta A: Mol Biomol Spectrosc., 2014, 123, 282-289; DOI:10.1016/j.saa.2013.12.072

12. Srinivasan P Kanagasekaran T and Gopalakrishnan R, Cryst Growth Des., 2008, 8(7), 2340-2345; DOI:10.1021/cg701143n

13. Karna S P, Prasad P N and Dupuis M, J Chem Phys., 1991, 94, 1171-1178; DOI:10.1063/1.460024

14. Woodford J N, Pauley M A and Wang C H, J Phys Chem A, 1997, 101(11), 19891992; DOI:10.1021/jp9639861

15. Singer K D, Sohn J E, King L A and Gordon H M, Katz H E and Dirk C W, J Opt Soc Am B, 1989, 6(7), 1339-1350; DOI:10.1364/JOSAB.6.001339

16. Hashimoto H, Okada Y, Fujimura H, Morioka M, Sugihara O, Okamoto N and Matsushima R, Jpn J Appl Phys., 1997, 36, 6754-6760

17. Bouchouit K, Essaidi Z, Abed S, Migalska-Zalas A, Derkowska B, Benali-Cherif N, Mihaly M, Meghea A, and Sahraoui B, Chem Phys Lett., 2008, 455(4-6), 270-274.

18. Gubler U, Concilio S, Bosshard C, Biaggio I, Günter P, Martin R E, Edelmann M J, Wytko J A and Diederich F, Appl Phys Lett., 2002, 81(13), 2322-2324; DOI:10.1063/1.1507834

19. Martin R E, Wytko J A, Diederich F, Boudon C, Gisselbrecht J P and Gross M, Helve Chim Acta, 1999, 82(9), 1470-1485; DOI:10.1002/(SICI)15222675(19990908)82:9<1470::AID-HLCA1470>3.0.CO;2-N

20. Kavitha E, Sandaraganesan N and Sebastian S, Indian J Pure Appl Phys., 2010, 48, 20-30.

21. Kim K H, Han Y K and Jung J, Theor Chem Acc., 2005, 113, 233-237; DOI:10.1007/s00214-005-0630-7 
22. Pearson R G, J Chem Sci., 2005, 117(5), 369-377; DOI:10.1007/BF02708340

23. Chattaraj P K and Maiti B, J Am Chem Soc., 2003, 125(9), 2705-2710; DOI:10.1021/ja0276063

24. Parr P G and Pearson R G, J Am Chem Soc., 1983, 105(26), 7512-7516; DOI:10.1021/ja00364a005

25. Mebi A C, J Chem Sci., 2011, 123(5), 727-731; DOI:10.1007/s12039-011-0131-2

26. Soscún H, Castellano O, Bermúdez Y, Toro-Mendoza C, Marcano A and Alvarado Y, J Mol Struct (Theochem) , 2002 , 592(1), 19-28; DOI:10.1016/S0166-1280(02)00222-1

27. Thanthiriwatte K S and De Silva K M N, J Mol Struct (Theochem), 2002, 617(1-3), 169-175; DOI:10.1016/S0166-1280(02)00419-0 